\title{
Remarks on Frobenius groups
}

\author{
Liguo He, Yubing Cao \\ Dept. of Math., Shenyang University of Technology \\ Shenyang, 110870, PR China
}

\begin{abstract}
Let the finite group $G$ act transitively and non-regularly on a finite set $\Omega$ whose cardinality $|\Omega|$ is greater than one. Use $N$ to denote the full set of fixed-point-free elements of $G$ acting on $\Omega$ along with the identity element. Write $H$ to denote the stabilizer of some $\alpha \in \Omega$ in $G$. In the note, it is proved that the subset $N$ is a subgroup of $G$ if and only if $G$ is a Frobenius group. It is also proved $G=\langle N\rangle H$, where $\langle N\rangle$ is the subgroup of $G$ generated by $N$.
\end{abstract}

Keywords- finite group, Frobenius group, permutation group

\section{INTRODUCTION}

Finite group $G$ is a transitive permutation group acting on a set $\Omega$, where $|\Omega|>1$. Recall that an element $g$ of $\mathrm{G}$ is a derangement if $g$ acts fixed-point-freely on $\Omega$. Let $N$ be the subset of $G$ consisting of all derangements together with the identity, so $N$ is clearly a normal subset of $G$, but it need not be a subgroup in general. We refer to $N$ as the derangement kernel of $G$. Observe that $G$ is the union of the derangement kernel $N$ together with all of point stabilizers, which are conjugate in $\mathrm{G}$, hence $|N|>1$. Recall that a transitive action of $\mathrm{G}$ on $\Omega$ is said to be a Frobenius action if every point stabilizer is nontrivial but the intersection of any two point stabilizers is trivial. A group $G$ is called a Frobenius group when it has a Frobenius action on some set $\Omega$ whose cardinality is greater than one. A celebrated theorem of Frobenius asserts that if $G$ is a Frobenius group, then its derangement kernel $N$ is a proper subgroup of $G$ (5), Theorem 7.2]), and in that case $N$ is called the Frobenius kernel. In 6, it is proved that if all elements in $N$ are involutions,then $N$ is an elementary abelian 2-group such that either $G=N$ or $G$ is a Frobenius group with kernel $N$. In this note, we show that if the derangement kernel $N$ is a proper subgroup, then the action of $\mathrm{G}$ on $\Omega$ is of Frobenius. When $N$ is a subgroup, it is easy to prove $G=N H$, where $H$ is a point stabilizer in $G$. In fact, there are other conditions to guarantee $G=N H$. For example, we show that $G=N H$ when $\mathrm{G}$ is 2 -transitive on $\Omega$ (Proposition 4). Also we show that it is always true that $G=\langle N\rangle H$, where $\langle N\rangle$ is the subgroup of $G$ generated by $N$ (Theorem 3). We even guess that $G=N H$ whenever $G$ has a transitive action on $\Omega$. However, we can neither prove the claim nor give a counterexample. Under only the hypothesis that $G$ acts transitively and non-regularly on $\Omega$, the subset $N$ is not generally a subgroup of $G$. We prove that $N$ is a group if and only if $G$ is Frobenius group (Theorem 1).

We mention that Frobenius groups paly a prominent role in the theory of finite groups, they usually act as either a starting point or a reduced goal (by the minimal counterexample argument) when investigating some problems of group theory, for example, see [1, 2, 8.

Unless otherwise stated, the notation and terminology is standard, as presented in [5].

Liguo $\mathrm{He} 1$ would like to take this opportunity to thank the NSF of China (Grant No.12171058) for its support.

\section{RESUlts}

The following result indeed shows that the derangement kernel $N$ is a group exactly when $G$ is a Frobenius group or a regular group. It is clear that $|G|=|N|=|\Omega|$ when $G$ is a regular group on $\Omega$.

Theorem 1. Let $G$ be a transitive and non-regular group acting on $\Omega$ with the derangement kernel $N$, then $|\Omega| \leq|N|$. Furthermore, the following statements are equivalent.

1) The action of $G$ on $\Omega$ is Frobenius.

2) The set $N$ is a subgroup of $G$.

3) The equality $|\Omega|=|N|$ holds.

Proof. Write $H$ for $C_{G}\left(\alpha_{1}\right)$. Since $G$ acts transitively on $\Omega$, it follows that $|\Omega|=n=|G: H|$ and $G=N \cup$ $C_{G}\left(\alpha_{1}\right) \cup C_{G}\left(\alpha_{2}\right) \cup \cdots \cup C_{G}\left(\alpha_{n}\right)$, then we may deduce that $|G| \leq|N|+n(|H|-1)$. Thus we get that

$$
|G| \leq|N|+n|H|-n=|N|+|G: H||H|-n,
$$

hence $|\Omega|=n \leq|N|$, as desired.

Now assume part 1 . Then the derangement kernel $N$ is just the Frobenius kernel, and Frobenius' theorem (4,

\footnotetext{
${ }^{1}$ Corresponding author, helg-lxy@sut.edu.cn
} 
Satz V.7.6] or [5, Theorem 7.2]) yields that $N$ is a group, part 2 follows.

Assuming part 2 we deduce that $|\Omega|=\left|\alpha_{1}^{G}\right|=$ $\left|\alpha_{1}^{C_{G}\left(\alpha_{1}\right) N}\right|=\left|\alpha_{1}^{N}\right|=\left|N: C_{N}\left(\alpha_{1}\right)\right|=|N|$, hence $|N|=|\Omega|$, part 3 follows.

Finally assume part 3 . We have $|\Omega|=|G: H|$, so $|G|=|\Omega||H|$. We are assuming that $|N|=|\Omega|=n$, and thus $|G|=|N||H|$. By the definition of $N$, we have $G=N \cup \bigcup_{1 \leq i \leq n} C_{G}\left(\alpha_{i}\right)$, so if we write $C_{i}=C_{G}\left(\alpha_{i}\right)-1$, we have $G=N \cup \bigcup_{1 \leq i \leq n} C_{i}$. Now, $\left|C_{i}\right|=|H|-1$ and by assumption, $|N|=n$, and thus we have $|N||H|=|G|=$ $\left|N \cup \bigcup_{1<i<n} C_{i}\right| \leq|N|+\sum_{1<i<n}\left|C_{i}\right|=|N|+n(|H|-1)=$ $|N|+|\bar{N}|(|H|-1)=|N||\bar{H}|$. Equality holds, and thus the union is disjoint. Then $C_{G}\left(\alpha_{i}\right) \cap C_{G}\left(\alpha_{j}\right)=1$ when $i$ and $j$ are different, and thus by definition, the action of $\mathrm{G}$ on $\Omega$ is Frobenius. The proof is finished.

The following consequence may be regarded as a slight improvement of Frattini argument (see [7, Theorem 2.1.4], for example).

Lemma 2. Let $G$ act transitively on the set $\Omega$ where $|\Omega|>1, H=C_{G}(\alpha)$ for $\alpha \in \Omega$ and $N$ a subset of $G$. Then $G=H N$ if and only if $\alpha^{N}=\Omega$, where $\alpha^{N}=\left\{\alpha^{n} \mid\right.$ $n \in N\}$.

Proof. If $G=H N$, then $\Omega=\alpha^{G}=\alpha^{H N}=\alpha^{N}$, as wanted. For $g \in G$, if $\alpha^{g}=\beta$ for $\beta \in \Omega$, then since $\alpha^{N}=\Omega$, there exists some $n \in N$ such that $\alpha^{n}=\beta$, thus $\alpha^{g n^{-1}}=\alpha$, so $g n^{-1} \in H$, hence $g \in H N$, and so $G=H N$, as desired.

Theorem 3. Let $G$ be a transitive group acting on $\Omega$ with the derangement kernel $N$ and $H=C_{G}\left(\alpha_{1}\right)$. Then the subgroup $\langle N\rangle$ is transitive on $\Omega$ and $\langle N\rangle H=G$. Furthermore, if $N H$ is subgroup, then $N H=G$.

Proof. Since $N$ is a normal subset, it follows that $\langle N\rangle$ is a normal subgroup, and thus $\langle N\rangle H$ is a subgroup that contains $N$. Now $\bigcup_{g \in G}(\langle N\rangle H)^{g}$ contains $N$ and all conjugates of $H$, and since $G$ is the union of $N$ and the conjugates of $H$, it follows that $\bigcup_{g \in G}(\langle N\rangle H)^{g}=G$. But it is a fact that if the union of all conjugates of some subgroup of a group is the whole group, then the subgroup must be the whole group. We have $G=\langle N\rangle H=H\langle N\rangle$. By Lemma 2, all $\alpha_{i}$ are in the $\langle N\rangle$-orbit containing $\alpha_{1}$, and thus $\langle N\rangle$ acts transitively. Finally, suppose $N H$ is a subgroup. Then $N H$ contains both $\langle N\rangle$ and $H$, so it contains $\langle N\rangle H=G$, and thus $N H=G$.

Observe that $G$ may be expressible as $G=N H$ even though $N$ is not a subgroup, as shown in the following consequence.

Proposition 4. Let $G$ act on the set $\Omega=$ $\left\{\alpha_{1}, \alpha_{2}, \cdots, \alpha_{n}\right\}$ with the derangement kernel $N$ and $H=C_{G}\left(\alpha_{1}\right), n>1$. If the action is 2-transitive, then $G=N H$.

Proof. Let $g \in G-H$ and $\alpha_{1}^{g}=\alpha_{i}$. Pick $1 \neq z \in N$ and let $\alpha_{1}^{z}=\alpha_{j}$. By the 2-transitivity, we know that $H$ acts transitively on the difference set $\Omega-\left\{\alpha_{1}\right\}$, and so there exists $h \in H$ such that $\alpha_{j}^{h}=\alpha_{i}$, then $\alpha_{1}^{z h g^{-1}}=\alpha_{1}$, which implies $z h g^{-1} \in H$ and so $g \in H N H$. Because $H N H=N H H=N H$, it follows $g \in N H$. We therefore conclude $G=N H$, as desired.

It is known that Symmetric group $S_{n}$ and Alternating group $A_{n}$ are 2 -transitive when $n \geq 4$. Thus they have the above product form.

For the alternating group $A_{5}$ of degree 5 , we may get via GAP (3]) that

$N=\{(),(1,5,4,3,2),(1,4,2,5,3),(1,3,5,2,4)$, $(1,2,3,4,5),(1,4,5,3,2),(1,2,4,3,5),(1,5,3,2,4)$, $(1,4,5,2,3),(1,5,4,2,3),(1,3,4,5,2),(1,5,3,4,2)$, $(1,3,2,4,5),(1,3,2,5,4),(1,2,4,5,3),(1,5,2,3,4)$, $(1,2,5,4,3),(1,4,3,2,5),(1,2,3,5,4),(1,4,3,5,2)$, $(1,3,4,2,5),(1,5,2,4,3),(1,4,2,3,5),(1,3,5,4,2)$, $(1,2,5,3,4)\}$, and its subset

$$
\{(),(1,5,4,3,2),(1,4,2,5,3),(1,3,5,2,4),(1,2,3,4,5)\}
$$

is actually a right transversal for $A_{4}$ in $A_{5}$, thus we achieve that $A_{5}=A_{4} N=N A_{4}$ (as $N$ is a normal subset). As $(1,4,5,3,2) *(1,5,4,3,2)=(1,3)(2,5) \notin N$, we see that $N$ is not a group. (The nonabelian simple group $A_{5}$ has a proper normal subset $N$ and a nontrivial factorization form $A_{5}=N A_{4}$. This is really an interesting thing! ) For $A_{6}$, we may also verify via GAP ([3]) that $A_{6}=A_{5} N=N A_{5}$, where $N$ is the derangement kernel of $A_{5}$.

\section{REFERENCES}

[1] R. Brown, Frobenius groups and classical maximal orders, Mem. Amer. Math. Soc., 2001, 717

[2] D.G. Costanzo, M.L. Lewis, The cyclic graph of a 2Frobenius group, arXive: 2103.15574v1[mathGR], 20 Mar 2021

[3] The GAP Group, GAP - Groups, algorithms, and programming, Version 4.7.5, http://www.gapsystem.org, 2014

[4] B. Huppert, Endliche Gruppen I, Springer-Verlag, Berlin-Heidelberg-New York, 1967

[5] I.M. Isaacs, Character Theory of Finite Groups, Academic Press, New York, 1976

[6] I. M. Isaacs, T. M. Keller, M.L. Lewis, Transitive permutation groups in which all derangements are involutions, Pure Appl. Algebra, 2006, 207: 717-724

[7] H. Kurzweil, B. Stellmacher, The Theory of Finite Groups: an Introduction, Springer-Verlag New York, 2004

[8] J. Maccrron, Frobenius groups with perfect order classes, arXive: 2103.00425v1[mathGR], 28 Feb 2021

\section{Creative Commons Attribution License 4.0 (Attribution 4.0 International, CC BY 4.0)}

This article is published under the terms of the Creative Commons Attribution License 4.0 https://creativecommons.org/licenses/by/4.0/deed.en US 